\title{
The Suzaku Hard X-ray Survey on the Galactic Center Region
}

Takeshi Go Tsuru*1, H. Uchiyama, M. Nobukawa, M. Sawada, S.G. Ryu, R. Fukuoka, K. Koyama

Department of Physics, Faculty of Science, Kyoto University, Kitashirakawa, Sakyo, Kyoto 606-8502, JAPAN

${ }^{1}$ E-mail: tsuruecr.scphys.kyoto-u.ac.jp

Diffuse X-rays from the Galactic center (GC) region were found to exhibit many K-shell lines from iron and nickel atoms in the $6-9 \mathrm{keV}$ band. The strong emission lines seen in the spectrum are neutral iron $\mathrm{K} \alpha$ at $6.4 \mathrm{keV}$, He-like iron $\mathrm{K} \alpha$ at $6.7 \mathrm{keV}, \mathrm{H}-$ like iron $\mathrm{Ly} \alpha$ at $6.9 \mathrm{keV}$, and He-like iron $\mathrm{K} \beta$ at $7.8 \mathrm{keV}$. Among them, the $6.4 \mathrm{keV}$ emission line is a probe of non-thermal phenomena. We have detected strong $6.4 \mathrm{keV}$ emission in several giant molecular clouds, some of which were newly discovered by Suzaku. All the spectra exhibit large equivalent widths of 1-2 $\mathrm{keV}$ and absorption columns of $2-10 \times 10^{23} \mathrm{H} \mathrm{cm}^{-2}$. We found time variability of diffuse $6.4 \mathrm{keV}$ emission in the Sgr B2 region comparing the maps and spectra obtained from 1994 to 2005 with ASCA, Chandra, XMM-Newton and Suzaku. We also report discovery of K $\alpha$ lines of neutral argon, calcium, chrome, and manganese atoms in the Sgr A region. We show that the equivalent width of the $6.4 \mathrm{keV}$ emission line detected in X-ray faint region against the $6.4 \mathrm{keV}$ associated continuum (power-law component) is $\sim 800 \mathrm{eV}$. These features are naturally explained by the X-ray reflection nebula scenario rather than the low energy cosmic-ray electrons scenario. On the other hand, a $6.4 \mathrm{keV}$ clump, G 0.162-0.217, discovered at the south end of the Radio Arc has a small equivalent width of $6.4 \mathrm{keV}$ emission line of $\sim 200 \mathrm{eV}$. The Radio Arc is a site of relativistic electrons. Thus, it is conceivable that the X-rays of G $0.162-0.217$ are due to low energy cosmic-ray electrons

The Extreme sky: Sampling the Universe above $10 \mathrm{keV}$ - extremesky2009,

October 13-17, 2009

Otranto (Lecce) Italy

\footnotetext{
${ }^{*}$ Speaker.
} 


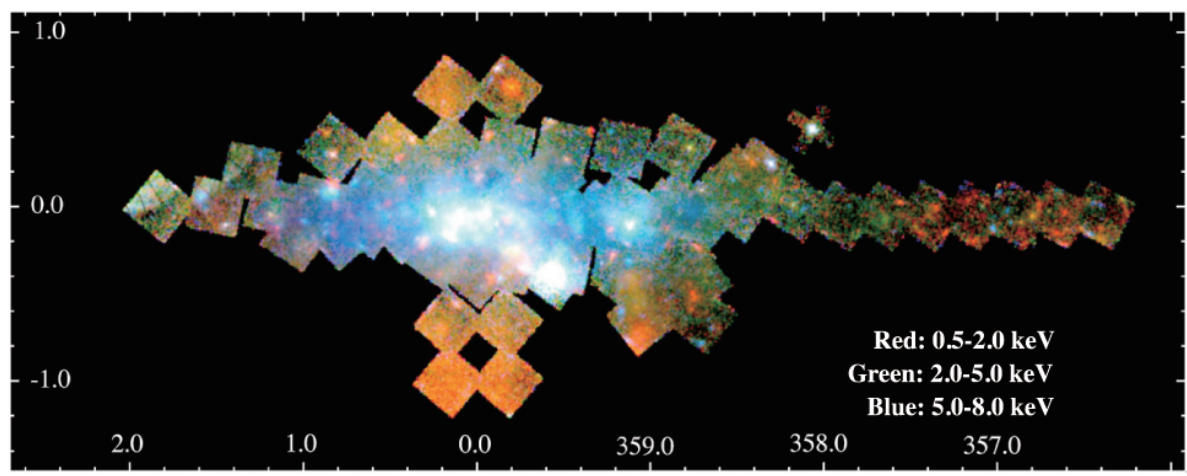

Figure 1: The X-ray image of the Galactic center region obtained with the Suzaku XIS. The colors indicate X-ray energy bands - red (0.5-2.0 keV), green (2.0-5.0 keV), and blue (5.0-8.0 keV).

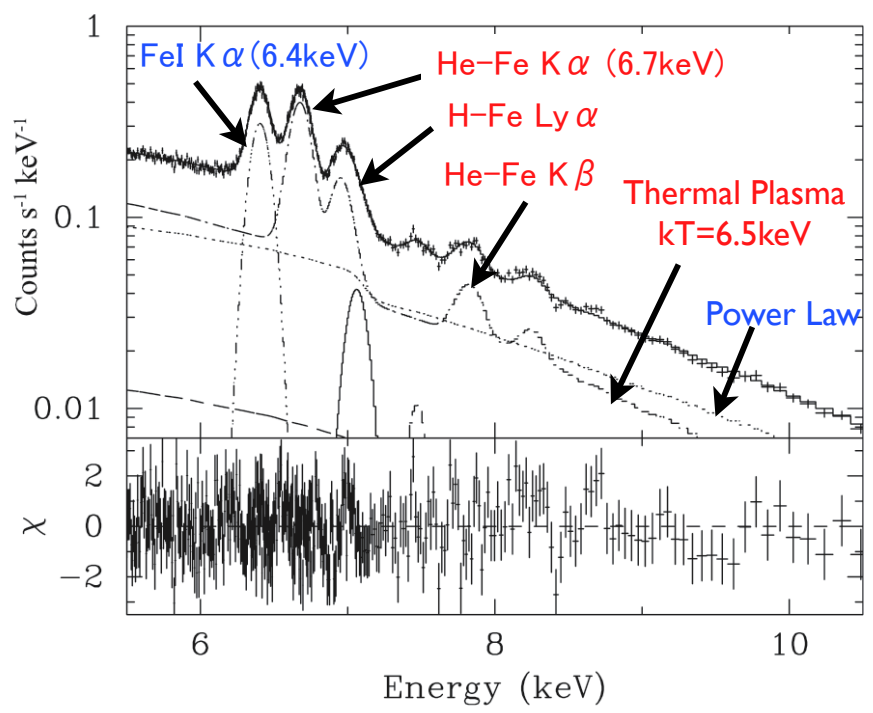

Figure 2: The averaged spectrum of the Sgr A region in the 5.5-11.5 keV band taken with the Suzaku XIS [1]. The spectrum is fitted with a model of a collisional ionization equilibrium plasma plus a power-law with three Gaussian lines and an iron absorption edge.

\section{Introduction}

The X-ray Imaging Spectrometer (XIS) on board the Suzaku satellite is the X-ray CCD camera system which has the large effective area, the low and the stable non X-ray background in the 0.3$12 \mathrm{keV}$ band. The diffuse hard X-ray emission from the Galactic center region, particularly in the iron K-shell band, is the best target for Suzaku. We have been making survey observation as a Suzaku key project. We have finished about 60 pointings with a total exposure time of $\sim 3000 \mathrm{ksec}$ and published 22 papers so far (Figure 1). Figure 2 shows an excellent spectrum of the Sgr A region [1]. The results from the key project can make a Suzaku legacy in the X-ray astronomy.

The spectrum of the diffuse X-rays from the Galactic center region exhibits many K-shell lines from iron and nickel atoms in the 6-9 keV band (Figure 2). The strong emission lines seen in the spectrum are neutral iron $\mathrm{K} \alpha$ at $6.4 \mathrm{keV}$, He-like iron $\mathrm{K} \alpha$ at $6.7 \mathrm{keV}$, $\mathrm{H}$-like iron $\mathrm{Ly} \alpha$ at $6.9 \mathrm{keV}$ and 

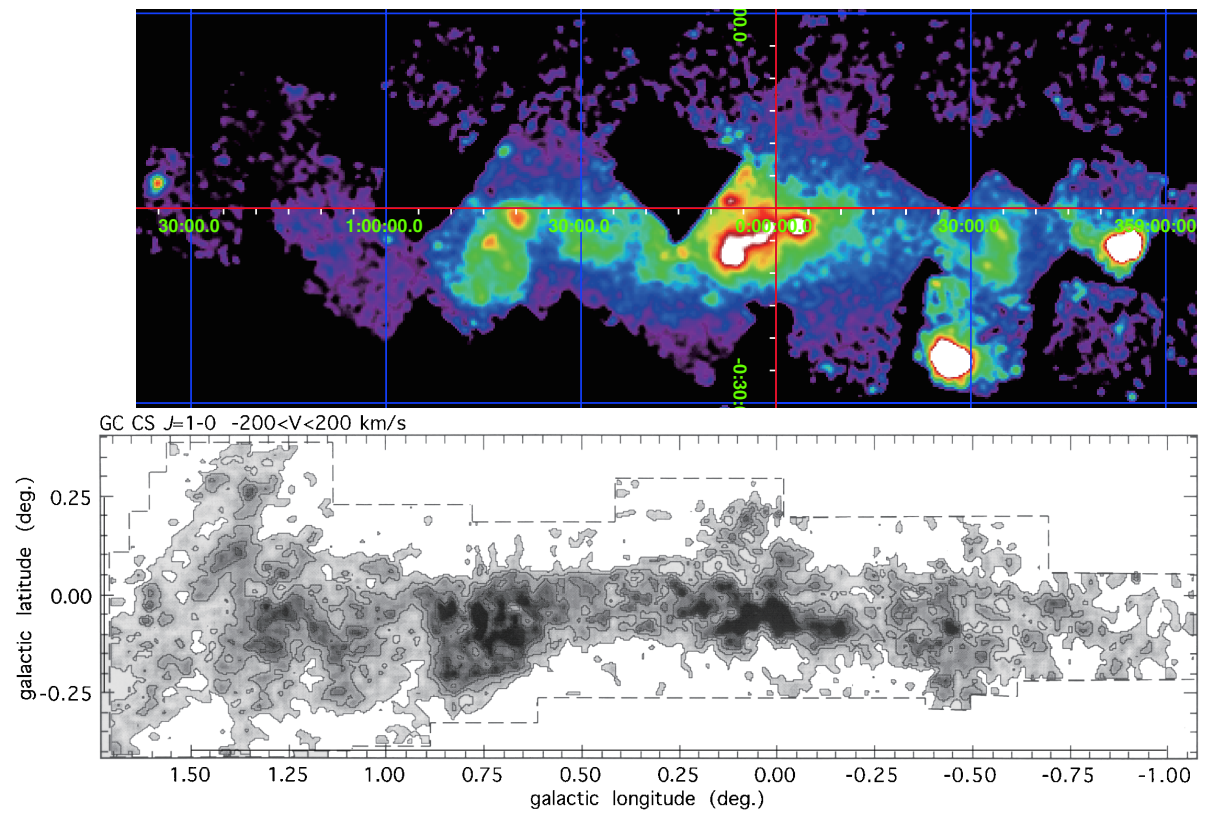

Figure 3: The maps of the $6.4 \mathrm{keV}$ line band (top) and the velocity-integrated carbon monosulfide line (bottom) [2].

He-like iron $\mathrm{K} \beta$ at $7.8 \mathrm{keV}$. We determined the electron temperature from the flux ratio of $\mathrm{K} \beta$ and $\mathrm{K} \alpha$ emission lines from He-like iron [1]. The ionization temperature was obtained from the fluxes of the emission lines of $\mathrm{H}$-like iron Ly $\alpha$ and He-like iron $\mathrm{K} \alpha$. Both temperatures are $k T \sim 6.5 \mathrm{keV}$. Thus, we can safely fix the continuum shape of the thermal component at the temperature of $k T=$ $6.5 \mathrm{keV}$. An additional power-law component with the photon index $\Gamma=1.4_{-0.7}^{+0.5}$ is necessary to fit the observed continuum spectrum [1]. The power-law component may have a non-thermal origin and will dominate in the X-ray spectrum above $10 \mathrm{keV}$. The spectral index is similar to those of $\mathrm{X}$-ray spectra in the $6.4 \mathrm{keV}$ clouds. It indicates that the $6.4 \mathrm{keV}$ emission line is related to the power-law component and can be a probe of the non-thermal phenomena.

\section{The 6.4 keV line emission from Giant Molecular Clouds}

Figure 3 shows the maps of the $6.4 \mathrm{keV}$ line and the carbon monosulfide line which traces molecular clouds [2]. The $6.4 \mathrm{keV}$ emission shows a clumpy distribution while the $6.7 \mathrm{keV}$ emission has few distinctive structures. Figure 3 indicates that the $6.4 \mathrm{keV}$ line emission generally traces the distribution of the carbon monosulfide line emission. It suggests that the iron K-shell fluorescence line emission occurs in the molecular clouds.

Two models have been proposed for the origin of the $6.4 \mathrm{keV}$ emission line. One is the Xray photo-ionization by external X-ray sources (the XRN scenario) [3, 4, 5]. Since no irradiating source capable of powering the $6.4 \mathrm{keV}$ line is found, Koyama et al. (1996) proposed a scenario of a past X-ray outburst of the super-massive black hole at Sgr A*. The other is the inner-shell ionization by the impact of low energy cosmic-ray electrons (the LECRe scenario) 6].

The power-law component is the Thomson scattered radiation of the incident X-rays in the $\mathrm{XRN}$ scenario, or the bremsstrahlung in the LECRe one. The expected X-ray spectrum is different 


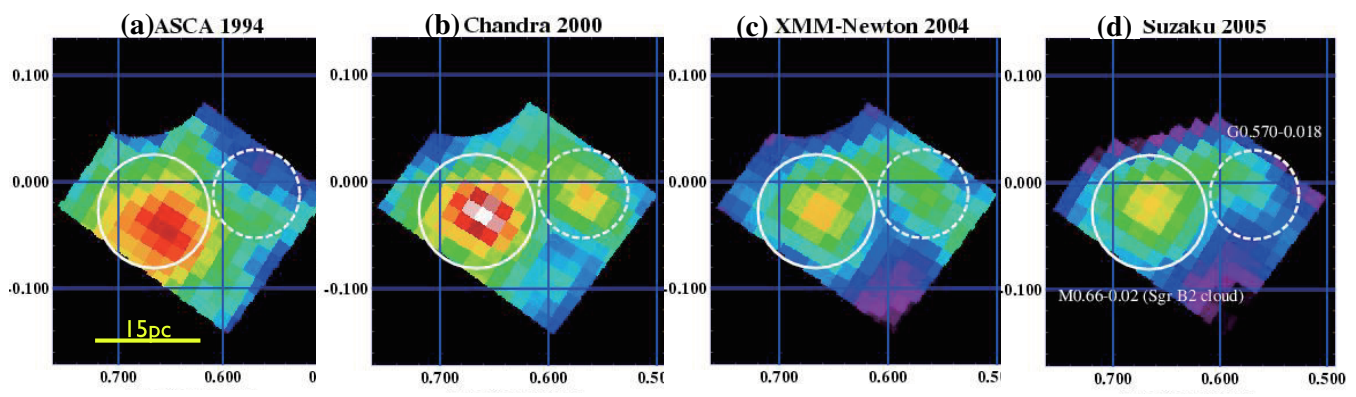

Figure 4: Surface brightness maps at the energy band of 6.0-7.0 keV including iron $\mathrm{K}$ lines obtained with (a) ASCA SIS in 1994, (b) Chandra ACIS-I in 2000, (c) XMM-Newton MOS and PN in 2004, and (d) Suzaku XIS in 2005 [14. Pixel size is 50" $\times 50$ ” in each case.

between the two scenarios. The $6.4 \mathrm{keV}$ line is expected to have a larger equivalent width in the $\mathrm{X}$-ray reflection $(\sim 1 \mathrm{keV})$ than that in the LECRe $(\sim 0.3 \mathrm{keV})$. Hard X-rays photo-ionizing iron can reach a deep portion of a molecular cloud, but electrons easily stop at the surface due to the ionization loss. Thus, larger absorption column is expected in the XRN model than in the LECRe one.

We have detected several $6.4 \mathrm{keV}$ clumps so far, some of which were newly discovered by Suzaku [9, 10, 11, 12]. All the spectra have large equivalent widths of 1-2 keV and absorption columns of $2-10 \times 10^{23} \mathrm{H} \mathrm{cm}^{-2}$. These features are naturally explained by the XRN scenario rather than the LECRe one.

We found time variability of the diffuse $6.4 \mathrm{keV}$ emission in the Sgr B2 region [13, 14]. Figure 4 shows the maps of the line components in 6-7 keV from 1994 to 2005 . The two region marked with solid and dashed circles in Figure 4 became bright in 2000 but faded in 2004 and 2005. We analyzed the X-ray spectra of the observations, and found that the flux of the $6.7 \mathrm{keV}$ emission line had been constant through the decade observations. On the other hand, the flux of the $6.4 \mathrm{keV}$ emission line changed by a factor of two.

The decay time of the flux is about $10 \mathrm{yrs}$. The size of the $6.4 \mathrm{keV}$ clump resolved by Chandra is 10 lyrs. Electrons with the energy range of $10-100 \mathrm{keV}$, where the cross section of inner shell ionization of iron is maximum, are unable to travel 10 lyrs in $10 \mathrm{yrs}$. On the other hand, the XRN model explains this time variability.

\section{Discovery of $\mathrm{K} \alpha$ lines from Neutral Atoms}

We have shown the results from the neutral iron $\mathrm{K} \alpha$ emission line $(6.4 \mathrm{keV})$. How about the other elements? These would provide new information to constrain the origin of the neutral clumps in the GC region. Since the relative existences of the lighter elements are 10 to 100 times smaller than that of iron, we search the brightest $6.4 \mathrm{keV}$ clump located in the Sgr A region for their fluorescence lines [15]. Figure 5a shows the spectrum of the clump. We made a spectral fitting with a model of two temperature thermal plasma components plus a power-law, three Gaussians for the neutral iron $\mathrm{K} \alpha, \mathrm{K} \beta$, and nickel $\mathrm{K} \alpha$ emission lines. There remained residuals at the energies of $\mathrm{K}$ emission lines of the highly ionized chrome and manganese. Since the APEC model, the 

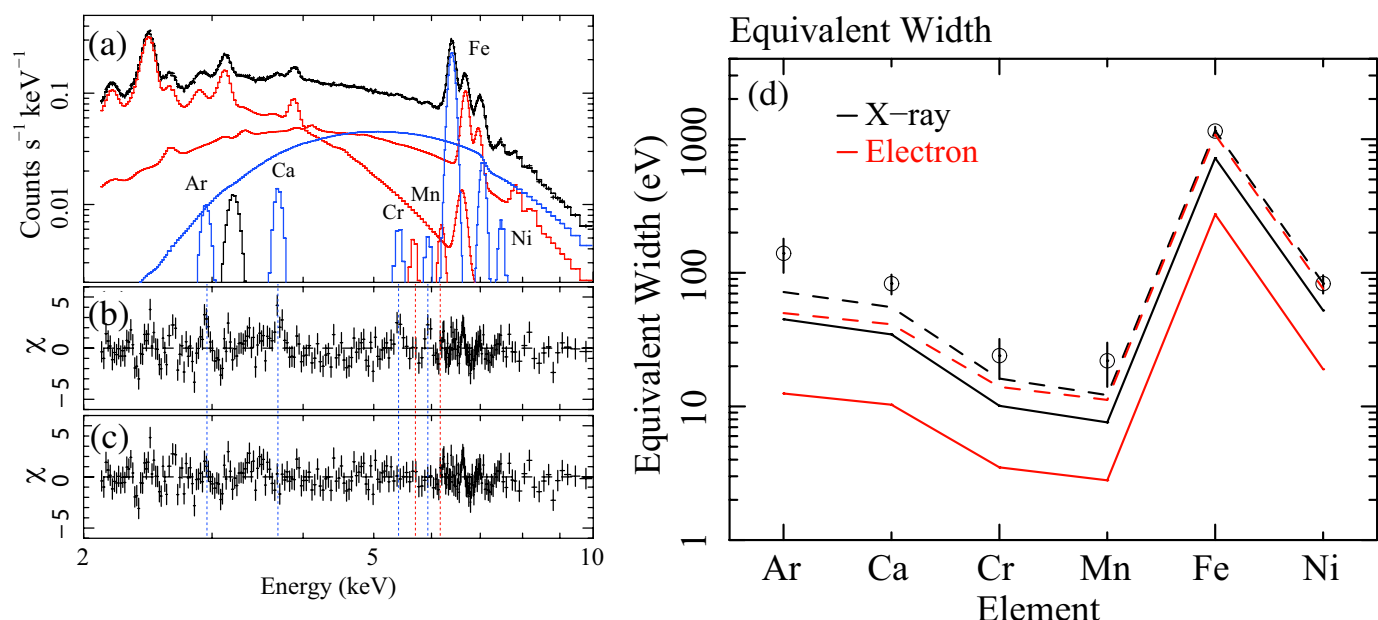

Figure 5: (a) Suzaku XIS-FI spectrum of the bright neutral clumps in the Sgr A region [15]. The figure is also plotted with the best fitted model consisting of 2- $k T$ APEC, He-like $\mathrm{Cr}$ and $\mathrm{Mn} \mathrm{K \alpha}$ lines (red), a power-law, neutral $\mathrm{Ar}, \mathrm{Ca}, \mathrm{Cr}, \mathrm{Mn}, \mathrm{Fe}, \mathrm{Ni} \mathrm{K} \alpha$ and iron $\mathrm{K} \beta$ emission lines (blue). (b) Residual of the fitting with a model of 2-kT APEC, He-like $\mathrm{Cr}$ and $\mathrm{Mn} \mathrm{K} \alpha$ lines a power-law, and neutral lines of Fe I K $\alpha, \mathrm{K} \beta$, Ni I K $\alpha$. (c) Same as (b) but the neutral Ar, Ca, Cr, and $\mathrm{Mn} \mathrm{K} \alpha$ lines are added. (d) Equivalent widths of $\mathrm{K} \alpha$ line of various neutral atoms. Black and red lines are the calculated value for the X-ray (XRN) and electron (LECRe) scenarios, respectively. The data points marked with the open circles are observed value in our work. Errors are estimated at the $90 \%$ confidence level. The black and red dashed lines are to guide eyes, which are XRN and LECRe scenarios in 1.6 solar and 4.0 solar abundances, respectively.

thermal plasma code used in the fitting, dose not contain the chrome and manganese K-lines, we added Gaussians for the lines. These lines were firstly detected from the Galactic center region. The temperatures of the plasmas are $1 \mathrm{keV}$ and $7 \mathrm{keV}$. The absorption column density for the plasma components is $7 \times 10^{22} \mathrm{H} \mathrm{cm}^{-2}$, which is typical for the sources in the Galactic center region. Abundances of the elements in the plasmas are $\sim 1.9$ solar for sulfur, argon, and calcium, $\sim 1.2$ solar for iron and $\sim 1.6$ for nickel. The power-law component has the index of $\sim 2$ and requires absorption column density of $1.7 \times 10^{23} \mathrm{H} \mathrm{cm}^{-2}$ which is significantly larger than that for the plasma components. There still remained four line-like residuals whose energies correspond to the neutral argon, calcium, chrome, and manganese $\mathrm{K} \alpha$ lines (Figure 5b). So, we added Gaussians modeling them to the spectral model and found that it improved the fitting significantly (Figure 55). We discovered the K-shell lines of neutral argon, calcium, chrome, manganese from the bright neutral clump toward the Sgr A.

Figure 5 $\mathrm{d}$ shows the equivalent widths of the neutral lines against the power-law component. In order to investigate the origin of the neutral lines, we calculated the equivalent widths in the two scenarios of the XRN and the LECRe using Geant 4. In this calculation, we assumed that density of the molecular cloud is uniform, the absorption column is $10^{23} \mathrm{H} \mathrm{cm}^{-2}$, and elemental abundances are solar. For the LECRe scenario, the metal abundances in the molecular cloud must be $\sim 4$ times larger than the solar value. The XRN scenario requires $\sim 1.6$ solar abundances. Since the molecular cloud may be formed by condensation of the ambient materials, the abundances should be similar to those in the plasma component in the GC region. The plasma components have the 


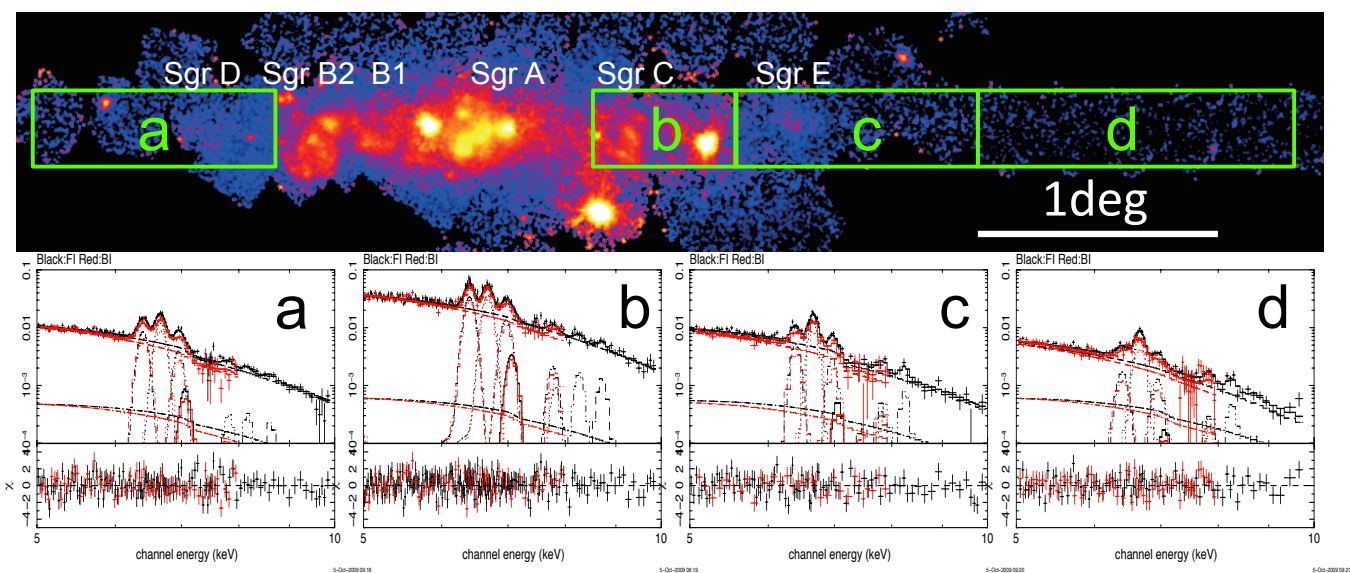

Figure 6: The spectra of the X-ray faint regions (bottom). The spectral extraction regions are overlaid on the $6.4 \mathrm{keV}$ map (top) 16 .

abundances of $\sim 1.2-1.9$, which agrees well with those required in the XRN scenario. Thus, we suggest that the $\mathrm{K} \alpha$ emission lines from neutral elements are due to the $\mathrm{X}$-ray reflection.

\section{The $6.4 \mathrm{keV}$ emission lines in $\mathrm{X}$-ray faint regions}

So far, we have shown the results from the X-ray bright clumps as the Sgr A and B2 regions. We now focus on X-ray faint region in this section. Figure 6 shows the regions investigated and their spectra. Significant $6.4 \mathrm{keV}$ emission lines are seen as those in the bright regions. Since the emission is so diffuse that the selection of the background region is difficult, we made a different approach.

Koyama et al. (2009) and Nakajima et al. (2009) showed that the Galactic center diffuse X-rays (GCDX) in the Sgr A and Sgr C regions is phenomenologically decomposed into the $6.7-\mathrm{keV}$ line plus an associated continuum (6.7-component) and the 6.4-keV line plus an associated continuum (6.4-component). The equivalent widths (EWs) of the $6.4 \mathrm{keV}\left(E W_{6.4}\right)$ and the $6.7 \mathrm{keV}\left(E W_{6.7}\right)$ lines for the Sgr A region

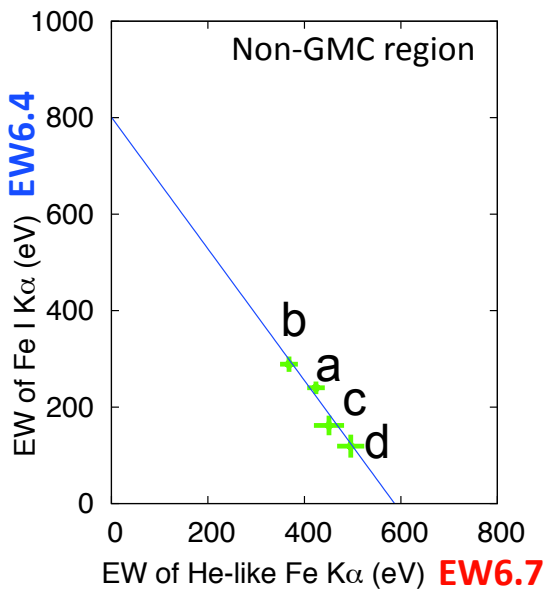

Figure 7: The relation between the equivalent widths of the $6.4 \mathrm{keV}$ and $6.7 \mathrm{keV}$ emission lines in the spectra of the X-ray faint regions (Figure 6) [16]. are given by the relation $E W_{6.7}+0.5( \pm 0.06) \times E W_{6.4}=0.62( \pm 0.07) \mathrm{keV}$ [8]. In the limit of $E W_{6.7} \rightarrow 0, E W_{6.4}$ in the 6.4-component was successfully estimated to be $1.2 \pm 0.2 \mathrm{keV}$.

We used the same method to decompose the GCDX in the X-ray faint regions [16]. Figure 7 shows the correlation plot between the observed EWs. This relation indicates that $E W_{6.4}$ in the 6.4component is estimated to be $\sim 800 \mathrm{eV}$ at $E W_{6.7} \rightarrow 0$. The values is significantly lower than the one expected in the XRN scenario but higher than that of the LECRe model. Further investigation is necessary. 

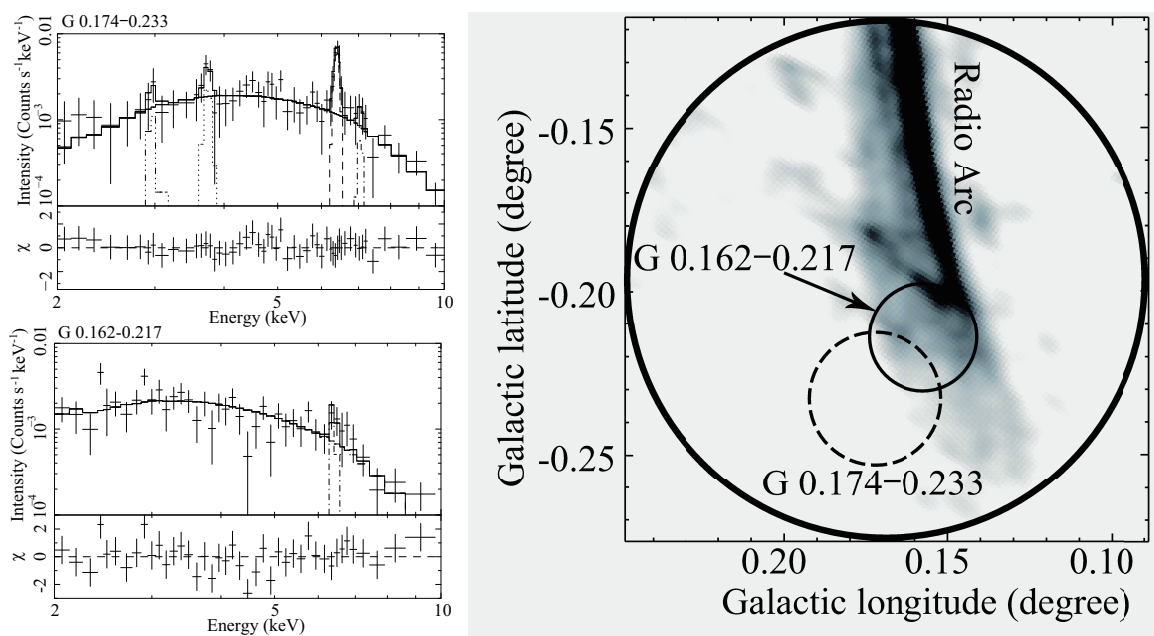

Figure 8: (left) Suzaku XIS (FI) spectra of G 0.174-233 and G 0.162-0.217 with the best-fit models of a power-law and Gaussian(s) [12]. The three emission lines seen in G 0.174-233 are neutral calcium, argon $\mathrm{K} \alpha$, iron $\mathrm{K} \alpha$ and $\mathrm{K} \beta$. The Gaussian shown in $\mathrm{G} 0.162-0.217$ is neutral iron K$\alpha$. (right) A $4.735 \mathrm{GHz}$ radio continuum map of the south end of the Radio Arc from the Very Large Array archive survey image. The Very Large Array field is shown by the thick solid circle. The positions of G 0.174-233 and G 0.162-217 are indicated by the dashed and thin-solid circles.

\section{G 0.162-0.127: a 6.4 keV clump due to LECRe}

Among the radio non-thermal filaments discovered in the Galactic center region [17], the most prominent one is the Radio Arc [18]. We found two $6.4 \mathrm{keV}$ clumps, G $0.174-0.233$ and G $0.162-0.217$, around the south end of the Radio arc [12]. The right panel of Figure 8 shows their positions with the close-up view of the Radio arc. The spectrum of G $0.174-0.233$ has prominent $\mathrm{K} \alpha$, emission lines of neutral iron and calcium (the left top panel of Figure 8). The EW of the iron $6.4 \mathrm{keV}$ emission line is $\sim 950 \mathrm{eV}$. Thus, the XRN scenario is favored for the origin of G $0.174-0.233$.

The the spectrum of G $0.162-0.217$ has significant $6.4 \mathrm{keV}$ emission line (the left bottom panel of Figure 8 ). The EW of the $6.4 \mathrm{keV}$ line of $\sim 200 \mathrm{eV}$ is $\sim 20 \%$ of the one expected by the XRN scenario but is consistent with the LECRe model $(\sim 300 \mathrm{eV})$. The right panel of Figure 8 indicates that G $0.162-0.217$ is located in the Radio Arc. Since the Radio Arc is a site of relativistic electrons, it may also include LECRe. Thus, it is quite conceivable that the $\mathrm{X}$-rays of G $0.162-0.217$ are due to the LECRe. Assuming the electron energy of 10-100 keV, thick condition for a target cloud and the radiation yield of $\sim 4 \times 10^{-5}$ due to bremsstrahlung, we obtained the electron energy density of $\sim 25 \mathrm{eV} \mathrm{cm}^{-3}$. The magnetic field is estimated to be $\sim 30 \mu \mathrm{G}$ in the condition of the equipartition.

\section{References}

[1] K. Koyama, Y. Hyodo, T. Inui, H. Nakajima, H. Matsumoto, T.G. Tsuru, et al. Iron and Nickel Line Diagnostics for the Galactic Center Diffuse Emission, PASJ 59, (2007) S245 
[2] M. Tsuboi, T. Handa and N. Ukita, Dense Molecular Clouds in the Galactic center region. I. Observations and Data, ApJS 129, (1999) 1

[3] K. Koyama, Y. Maeda, T. Sonobe, T. Takeshima, Y. Tanaka, S. Yamauchi, ASCA View of Our Galactic Center: Remains of Past Activities in X-Rays ?, PASJ 48, (1996) 249

[4] R. Sunyaev, E. Churazov Equivalent width, shape and proper motion of the iron fluorescent line emission from molecular clouds as an indicator of the illuminating source X-ray flux history, MNRAS 297, (1998) 1279

[5] S. Park, M.P. Muno, F.K. Baganoff, Y. Maeda, M. Morris, C. Howard, M.W. Bautz, G.P. Garmire, Characteristics of diffuse X-ray line emission within 20 parsecs of the Galactic center, ApJ 559, (2004) 603

[6] F. Yusef-Zadeh, C. Law, M. Wardle The origin of X-ray emission from a Galactic center molecular cloud: low-energy cosmic-ray electrons, ApJL 568 (2002) L121

[7] A. Valinia, V. Tatischeff, K. Arnaud, K. Ebisawa, R.Ramaty On the origin of the iron K line in the spectrum of the Galactic X-ray background, ApJ 543, (2000) 733

[8] K. Koyama, Y. Takikawa, Y. Hyodo, T. Inui, M. Nobukawa, H. Matsumoto, and T.G. Tsuru Spatial Distribution of the Galactic Center Diffuse X-Rays and the Spectra of the Brightest 6.4 keV Clumps, PASJ 61, (2009) S255

[9] H. Nakajima, T.G. Tsuru, M. Nobukawa, H. Matsumoto, K. Koyama, H. Murakami, A. Senda, $\mathrm{S}$. Yamauchi X-Ray Reflection Nebulae with Large Equivalent Widths of the Neutral Iron Ka Line in the Sagittarius C Region, PASJ 61, (2009) S233

[10] K. Koyama, T. Inui, Y. Hyodo, H. Matsumoto, T.G. Tsuru, Y. Maeda, H. Murakami, S. Yamauchi, S.E. Kissel, K.-W. Chan, Y. Soong Discoveries of Diffuse Iron Line Sources from the Sgr B Region, PASJ 59, (2007) S221

[11] M. Nobukawa, T.G. Tsuru, Y. Takikawa, Y. Hyodo, T. Inui, H. Nakajima, H. Matsumoto, K. Koyama, H. Murakami, S. Yamauchi, Suzaku Spectroscopy of an X-Ray Reflection Nebula and a New Supernova Remnant Candidate in the Sgr B1 Region, PASJ 60, (2008) S191

[12] R. Fukuoka, K. Koyama, S.G. Ryu, T.G. Tsuru Suzaku Observation Adjacent to the South End of the Radio Arc, PASJ 61 (2009) 593

[13] K. Koyama, T. Inui, H. Matsumoto, T.G. Tsuru A Time-Variable X-Ray Echo: Indications of a Past Flare of the Galactic-Center Black Hole, PASJ 60, (2008) S201

[14] T. Inui, K. Koyama, H. Matsumoto, T.G. Tsuru Time Variability of the Neutral Iron Lines from the Sagittarius B2 Region and Its Implication of a Past Outburst of Sagittarius A, PASJ 61 (2009) S241

[15] M. Nobukawa, K. Koyama, T.G. Tsuru, S.G. Ryu and V. Tatischeff, Discovery of K-shell Emission Lines of Neutral Atoms in the Galactic Center Region, PASJ (2010) in Printing

[16] H. Uchiyama Study of the Galactic Diffuse X-ray Emission with the Suzaku satellite Doctor Thesis, Kyoto University, (2010)

[17] T.N. LaRosa, M.E. Kassim, T.J.W.Lazio, S.D. Hyman A wide-field 90 centimeter VLA image of the Galactic center region, AJ 119 (2000) 207

[18] F. Yusef-Zadeh, M. Morris, D. Chance, Large, highly organized radio structures near the galactic centre, Nature 310 (1984) 557 